\title{
Differential approximation for some routing problems
}

\author{
Cristina Bazgan ${ }^{1}$, Refael Hassin ${ }^{2}$, Jérôme Monnot ${ }^{1}$ \\ 1 Université Paris Dauphine, LAMSADE, 75016 Paris, France \\ \{bazgan, monnot\}@lamsade.dauphine.fr \\ 2 Dep. of Statistics and Operations Research,Tel-Aviv University,Tel-Aviv 69978 \\ hassin@post.tau.ac.il
}

\begin{abstract}
We study vehicle routing problems with constraints on the distance traveled by each vehicle or on the number of vehicles. The objective is to minimize the total distance traveled by vehicles. We design constant differential approximation algorithms for some of these problems. In particular we obtain differential bounds: $\frac{1}{2}$ for METRIC 3VRP, $\frac{3}{5}$ for Metric $4 \mathrm{VRP}, \frac{2}{3}$ for Metric $k \mathrm{VRP}$ with $k \geq 5, \frac{1}{2}$ for the nonmetric case for any $k \geq 3$, and $\frac{1}{3}$ for CONSTRAINED VRP. We prove also that Min-Sum E $k$ TSP is $\frac{2}{3}$ differential approximable and has no differential approximation scheme, unless $\mathbf{P}=\mathbf{N P}$.
\end{abstract}

Keywords: differential ratio, approximation algorithm, VRP, TSP

\section{Introduction}

Vehicle routing problems that involve the periodic collection and delivery of goods and services as mail delivery or trash collection are of great practical importance. Simple variants of these real problems can be modeled naturally with graphs. Unfortunately even simple variants of vehicle routing problems are NP-hard. In this paper we consider approximation algorithms, and measure their efficiencies in two ways. One is the standard measure giving the ratio $\frac{a p x}{o p t}$, where opt and apx are the values of an optimal and approximate solution, respectively. The other measure is the differential measure, that compares the worst ratio of, on the one hand, the difference between the cost of the solution generated by the algorithm and the worst cost, and on the other hand, the difference between the optimal cost and the worst cost. Formally, the differential measure gives the ratio $\alpha=\frac{w o r-a p x}{w o r-o p t}$, where wor is the value of the optimal solution for the complementary problem. In [11], the measure $1-\alpha$ is considered and it is called there $z$-approximation. Justification for this measure can be found for example in $[1,5,18,11,14]$.

The main subject of this paper is differential approximation of the routing problems. In these problems $n$ customers have to be served by vehicles of limited capacity from a common depot. A solution consists of a set of routes, where each starts at the depot and returns there after visiting a subset of customers, such that each customer is visited exactly once. We refer to a problem as a VEHICLE 
ROUTING PROBLEM (VRP) if there is a constraint on the (possibly weighted) number of customers visited by a vehicle. This constraint reflects the assumption that the vehicle has a finite capacity and that it collects from the customers (or distributes among them) a commodity. The goal is to find a solution such that the total length of the routes is as small as possible. In other cases, the vehicle is just supposed to visit the customers, for example, in order to serve them. In such cases we refer to the problem as a TSP problem.

The problems that are considered here generalize the (undirected) TRAVELIng Salesman Problem (TSP). Differential approximation algorithms for the TSP are given by Hassin and Khuller [11] and Monnot [14]. We will sometimes use these algorithms to generate approximations for the problems of this paper. However, we note an important difference. In the TSP, adding a constant $k$ to all of the edge length does not affect the set of optimal solutions or the value of the differential ratio. The reason is that every solution contains exactly $n$ edges and therefore every solution value increases by exactly the same value, namely $n \cdot k$. In particular, this means that for the purpose of designing algorithms with bounded differential ratio, it doesn't matter whether $d$ is a metric or not (it can be made a metric by adding a suitable constant to the edge lengths). In contrast, in some of problems dealt with here, the number of edges used by a solution is not the same for every solution and therefore it may turn out, as we will see, that in some cases the metric version is easier to approximate.

It is easy to see that $2 \mathrm{VRP}$ is polynomial time solvable. For $k \geq 3$, METRIC $k$ VRP was proved NP-hard by Haimovich and Rinnooy Kan [8]. In [9], Haimovich, Rinnooy Kan and Stougie gave a $\frac{5}{2}-\frac{3}{2 k}$ standard approximation for Metric $k \mathrm{VRP}$. We study for the first time the differential approximability of $k$ VRP. More exactly we give a $\frac{1}{2}$ differential approximation for the non-metric case for any $k \geq 3$. We improve it to $\frac{3}{5}$ for METRIC $4 \mathrm{VRP}$ and to $\frac{2}{3}$ for METRIC $k \mathrm{VRP}$ with $k \geq 5$. An approximation lower bound of $\frac{2219}{2220}$ is given here for METRIC $n$ VRP with length 1 and 2 using a lower bound of $\operatorname{TSP}(1,2)[6]$.

Min-SuM E $k$ TSP is a generalization of TSP where we search to cover the customers by exactly $k$ vehicles such that the total length is minimum. Bellmore and Hong [3] showed that when we search for a solution with at most $k$ cycles then Min-Sum $k$ TSP is equivalent to TSP on an extended graph. As for MiN TSP, Min-Sum E $k$ TSP is differential equivalent to METrIC Min-Sum $k$ TSP or MAX-Sum EkTSP. We show in this paper that Metric Min-Sum E $k$ TSP is $\frac{2}{3}$ differential approximable and it has no differential approximation scheme unless $\mathbf{P}=\mathbf{N P}$.

The paper is organized as follows: In section 2 we give the necessary definitions. In section 3 we give a constant differential approximation algorithm for GENERAL $k$ VRP, and a better constant differential approximation for the metric case. In section 4 the main result is a constant differential approximation for Constrained VRP. In section 5 we show that Min-Sum E $k$ TSP is constant differential approximable and has no differential approximation scheme. 


\section{Terminology}

We first recall a few definitions about differential approximability. Given an instance $x$ of an optimization problem and a feasible solution $y$ of $x$, we denote by $\operatorname{val}(x, y)$ the value of the solution $y$, by opt $(x)$ the value of an optimal solution of $x$, and by $w o r(x)$ the value of a worst solution of $x$. The differential approximation ratio of $y$ is defined as $\delta(x, y)=\frac{|\operatorname{val}(x, y)-\operatorname{wor}(x)|}{|\operatorname{opt}(x)-\operatorname{wor}(x)|}$. This ratio measures how the value of an approximate solution $\operatorname{val}(x, y)$ is located in the interval between $\operatorname{opt}(x)$ and wor $(x)$. More exactly it is equivalent for a minimization problem to prove $\delta(x, y) \geq \varepsilon$ and $\operatorname{val}(x, y) \leq \varepsilon \operatorname{copt}(x)+(1-\varepsilon) \operatorname{wor}(x)$.

For a function $f, f(n)<1$, an algorithm is a differential $f(n)$-approximation algorithm for a problem $Q$ if, for any instance $x$ of $Q$, it returns a solution $y$ such that $\delta(x, y) \geq f(|x|)$. We say that an optimization problem is constant differential approximable if, for some constant $\delta<1$, there exists a polynomial time differential $\delta$-approximation algorithm for it. An optimization problem has a differential polynomial time approximation scheme if it has a polynomial time differential $(1-\varepsilon)$-approximation, for every constant $\varepsilon>0$. We say that two optimization problems are differential equivalent (reps., standard equivalent) if a differential $\delta$-approximation (reps., standard $\delta$-approximation) algorithm for one of them implies a differential $\delta$-approximation (reps., standard $\delta$-approximation) algorithm for the other one.

We consider in this paper several routing problems. The problems are defined on a complete undirected graph denoted $G=(V, E)$. The vertex set $V$ consists of a depot vertex 0 , and customer vertices $\{1, \ldots n\}$. There is also a function $d: E \rightarrow R$, where $d_{i, j} \geq 0$ denotes the length of edge $(i, j) \in E$. In the rest of the paper we call a such graph a complete valued graph. We refer to the version of the problem in which $d$ is assumed to satisfy the triangle inequality as the metric case. The output to the problems consists of simple cycles, $C_{1}, \ldots, C_{p}$, such that $V\left(C_{i}\right) \cap V\left(C_{j}\right)=\{0\}, \forall i \neq j$, and $\cup_{i=1}^{p} V\left(C_{i}\right)=V$. We call such a set of cycles a $p$-tour. We now describe the problems. For each one we specify the input, the problem's constraints, and the output.

$k \mathrm{VRP}$

Input: A complete valued graph.

Constraint: $\left|C_{j}\right| \leq k+1, j=1, \ldots, p$.

Output: A $p$-tour minimizing the total length of the cycles.

COnstrained VRP

Input: A complete valued graph and a metric $\ell: E \rightarrow R_{+}$, and $\lambda>0$.

Constraint: $\sum_{(i, j) \in C_{q}} \ell_{i, j} \leq \lambda, q=1, \ldots, p$.

Output: A $p$-tour minimizing the total length of the cycles. 


\section{$k$ WVRP}

Input: A complete valued graph and a function $w:\{1, \ldots, n\} \rightarrow R$ where $w_{i}$ denotes the weight of $i$.

Constraint: $\sum_{i \in C_{j}} w_{i} \leq k, j=1, \ldots, p$.

Output: A $p$-tour minimizing the total length of the cycles.

\section{Min-Sum E $k$ TSP}

Input: A complete valued graph.

Constraint: $p=k$.

Output: A $p$-tour minimizing the total length of the cycles.

For a problem $\mathrm{Q}$, we denote by $\mathrm{Q}(1,2)$ the version of $\mathrm{Q}$ where lengths are 1 and 2. We will use in this paper the following problems:

Min TSP PATH $(1,2)$ is the variant of Min TSP $(1,2)$ problem where in place of a tour we ask for a Hamiltonian path of minimum length. We can prove that Min TSP PATH $(1,2)$ where the graph induced by edges of weight 1 is Hamiltonian and cubic has no differential approximation scheme, unless $\mathbf{P}=\mathbf{N P}$. The result follows since Min TSP PATH $(1,2)$ on these instances has no standard approximation scheme, unless $\mathbf{P}=\mathbf{N P}$, Bazgan [2].

PARTITIONING INTO PATHS OF LENGTH $k$ ( $k \mathrm{PP})$ : Given a graph $G=(V, E)$ with $|V|=(k+1) q$, is there a partition of $V$ into $q$ disjoint sets $V_{1}, \ldots, V_{q}$ of $k+1$ vertices each, so that each subgraph induced by $V_{i}$ has an Hamiltonian path? 2PP have been proved NP-complete in [7] whereas, more generally, the NP-completeness of $k \mathrm{PP}$ is proved in Kirkpatrick and Hell [12] as a special case of $G$-PARTITION PROBLEM.

A binary 2-matching (also called 2-factor or cycle cover) is a subgraph in which each vertex of $V$ has a degree of exactly 2. A minimum binary 2-matching is one with minimum total edge weight. Hartvigsen [10] has shown how to compute a minimum binary 2-matching in $O\left(n^{3}\right)$ time. More generally, a binary $f$-matching, where $f$ is a vector of size $n+1$, is a subgraph in which each vertex $i$ of $V$ has a degree of exactly $f_{i}$. A minimum $f$-matching is one with minimum total edge weight and is computable in polynomial time, Cook et al. [4].

\section{$3 \quad k$ VRP}

$n \mathrm{VRP}$ is standard equivalent with TSP. So, when the distance is not metric and using the result of Sahni and Gonzalez [17] we deduce that $n$ VRP is not $2^{p(n)}$ standard approximable for any polynomial $p$, unless $\mathbf{P}=\mathbf{N P}$. In fact for any $k \geq 5$ constant the problem is as hard to approximate as $n \mathrm{VRP}$.

\subsection{General $k$ VRP}

When $d$ is a metric, the reduction of TSP to $n \mathrm{VRP}$ is straightforward, and it easily follows that computing opt is NP-hard. However, there is no such reduction between the corresponding maximization problems MAX TSP and MAX $n$ VRP leading to the conclusion that computing wor is also NP-hard. 
Proposition 1. Computing a worst solution for $k \mathrm{VRP}$ is NP-hard for any $k \geq$ 3 even if the distance function takes only two values.

Proof. We use a reduction from PARTITIONING INTO PATHS OF LENGTH $k(k \mathrm{PP})$. Let $G=(V, E)$ with $V=\{1, \ldots,(k+1) q\}$ be an instance of $k \mathrm{PP}$. We construct an instance of $k \mathrm{VRP}$ by the following way: we add the depot vertex 0 and set $d_{e}=3$ if $e \in E$ and $d_{e}=1$ otherwise. It is easy to verify that the answer to $k \mathrm{PP}$ is positive if and only if $w o r \geq q(3 k+2)$.

In the following we give a $\frac{1}{2}$ differential approximation for non-metric $k$ VRP. We apply ideas similar to those in Hassin and Khuller [11].

We first compute a lower bound $L B$. Then we generate a feasible solution for $G$ with value $a p x=L B+\delta_{1}$. Next, we generate another feasible solution of value $b a d=L B+\delta_{2}$ where $\delta_{2} \geq \delta_{1}$. This proves that the approximate solution with value apx is an $\alpha$-differential approximation where

$$
\alpha=\frac{w o r-a p x}{w o r-o p t} \geq \frac{b a d-a p x}{b a d-o p t} \geq \frac{\delta_{2}-\delta_{1}}{b a d-L B}=\frac{\delta_{2}-\delta_{1}}{\delta_{2}},
$$

since for a minimization problem $w o r \geq b a d \geq a p x \geq o p t \geq L B$. To generate $L B$ we replace 0 by a complete graph with a set $V_{0}$ of $2 n$ vertices and zero length edges. The distance between a vertex of $V_{0}$ and a vertex $i$ of $V \backslash V_{0}$ is the same as the distance between 0 and $i$. Denote the resulting graph by $G^{\prime}$. Compute in $G^{\prime}$ a minimum weight binary 2-matching $M$.

Lemma 1. Let $L B$ denote the weight of $M$. Then opt VRP $\geq L B$.

Theorem 1. $k \mathrm{VRP}$ is $\frac{1}{2}$ differential approximable.

Proof. We transform each cycle of $M$ into a cycle in $G$, replacing the vertices of $V_{0}$ by a single occurrence of the depot vertex 0 . We work on each cycle of $M$ separately. For each cycle we describe solutions $s o l_{1}, s o l_{2}$ and $s l_{3}$ such that $\delta\left(\operatorname{sol}_{1}\right)+\delta\left(\operatorname{sol}_{2}\right)=\delta\left(s o l_{3}\right)$. We define apx to be the VRP solution obtained by concatenating the shortest of $s_{1} l_{1}$ and $s_{2} l_{2}$ for every cycle, and we define bad similarly but using $\mathrm{sol}_{3}$. We obtain therefore that $\delta(b a d) \geq 2 \delta(a p x)$, and the theorem is proved by (1). In this proof, sol $_{3}$ will be always described by the cycles $(0,1,0), \ldots,(0, m, 0)$.

First, consider a cycle that does not contain the depot, and w.l.o.g. denote its vertices by $(1, \ldots, m)$. The construction depends on the parity of $m$.

Suppose that $m$ is even ( $m \neq 2$ since the 2 -matching is binary). Let sol $_{1}$ consist of the cycles $(0,1,2,0), \ldots,(0, m-1, m, 0)$. Let $s l_{2}$ consist of the cycles $(0, m, 1,0), \ldots,(0, m-2, m-1,0)$ (See Figure 1 for $m=6)$.

Suppose now that $m$ is odd. We modify $s_{0} l_{1}$ by choosing $(0, m-2, m-1, m)$ as the last cycle (see Figure 2). We modify $\operatorname{sol}_{2}$ by using $(0, m-1,0)$ as the last cycle.

Consider a cycle $(0,1, \ldots, m, 0)$ in $M$. If $m \leq k$ we don't change it. Otherwise:

If $m \equiv 0$ mod 3 then let $s o l_{1}$ consist of the cycles $(0,1,0),(0,2,3,0), \ldots,(0, m-$ $2,0),(0, m-1, m, 0)$. Let $s^{2} l_{2}$ consist of the cycles $(0, m, 1,2,0), \ldots,(0, m-3, m-$ $2, m-1,0)$. 


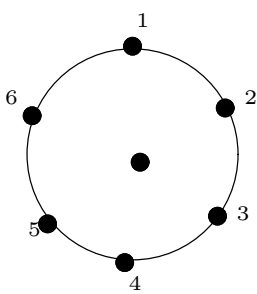

C

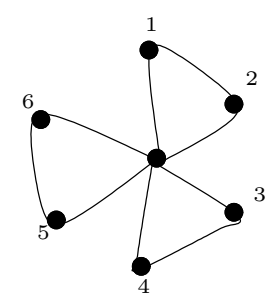

$\operatorname{sol}_{1}$

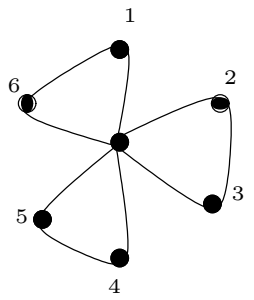

$\mathrm{sol}_{2}$

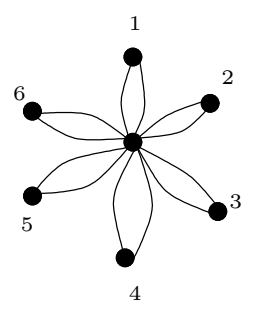

$\mathrm{sol}_{3}$

Fig. 1. $m=6$

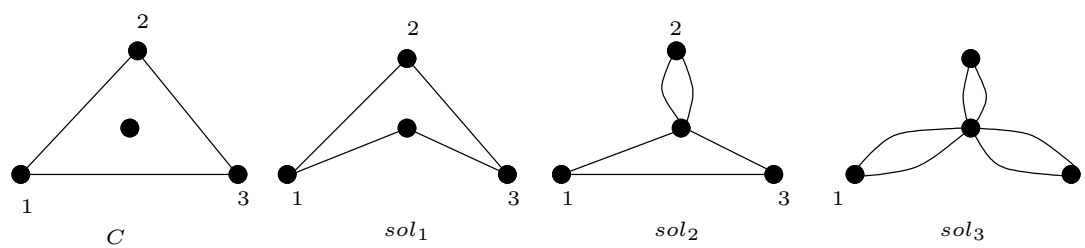

Fig. 2. $m=3$

If $m \equiv 1$ mod 3 then let $\operatorname{sol}_{1}$ consist of the cycles $(0,1,0),(0,2,3,0), \ldots,(0, m-$ $3,0),(0, m-2, m-1, m, 0)$. Let $\operatorname{sol}_{2}$ consist of the cycles $(0, m, 1,2,0), \ldots,(0, m-$ $4, m-3, m-2,0)$, and $(0, m-1,0)$.

If $m \equiv 2 \bmod 3$ then let $\operatorname{sol}_{1}$ consist of the cycles $(0,1,0),(0,2,3,0), \ldots,(0, m-$ $3, m-2,0),(0, m-1, m, 0)$. Let $s_{2} l_{2}$ consist of the cycles $(0, m, 1,2,0), \ldots,(0, m-$ $5, m-4, m-3,0)$, and $(0, m-2, m-1,0)$.

Let $\delta\left(s o l_{i}\right)$ denote the added cost of $s_{i} l_{i}$ with respect to the length of $C$. Since $M$ was computed to have a minimum cost, $\delta\left(\right.$ sol $\left._{i}\right) \geq 0$ and we have $\delta\left(\operatorname{sol}_{3}\right)=$ $\delta\left(\operatorname{sol}_{1}\right)+\delta\left(\operatorname{sol}_{2}\right)$ which complete the proof.

\subsection{Metric $\boldsymbol{k}$ VRP}

When $d$ is a metric, computing a worst solution becomes easy since any feasible cycle can be broken into two cycle without decrease the weight and then:

Lemma 2. or $_{V R P}=2 \sum_{i=1}^{n} d_{0, i}$

In Theorem 1 we have shown that $k \mathrm{VRP}$ is $\frac{1}{2}$ differential approximable. We now show that in the metric case, the same bound can be achieved by a simpler algorithm: we compute a minimum weight perfect matching $M$ on the subgraph induced by $\{1, \ldots, n\}$, if $n$ is even, or by $\{0,1, \ldots, n\}$ if $n$ is odd. We link each endpoint different of 0 of $M$ to the depot. It is easy to see that $o p t_{V R P} \geq 2 d(M)$ by walking around an optimum solution for $k \mathrm{VRP}$ and by shortcut it in order to obtain a Hamiltonian cycle. Using Lemma 2 and the construction of the 
approximate solution, we obtain: $a p x=d(M)+\sum_{i=1}^{n} d_{0, i} \leq \frac{1}{2}$ opt $t_{V P}+\frac{1}{2}$ wor $_{V R P}$ proving that the result is a $\frac{1}{2}$ differential approximation.

In Haimovich et al. [9], a $\frac{5}{2}-\frac{3}{2 k}$ standard approximation for METRIC $k$ VRP is obtained by reduction to METRIC TSP and using Christofides' algorithm. We proceed similarly for the differential case.

Theorem 2. METRIC $k \mathrm{VRP}$ is $\delta \cdot \frac{k-1}{k}$ differential approximable, where $\delta$ is the differential approximation ratio for METRIC TSP.

Proof. Our algorithm modifies the Optimal Tour Partitioning heuristic of Haimovich, Rinnooy Kan and Stougie [9]: first construct a tour $T$ on $V$ using the differential approximation algorithm for TSP of value $\operatorname{val}(T)$. W.l.o.g., assume that this tour is described by the sequence $(0,1, \ldots, n, 0)$. We produce $k$ solutions $s_{i}$ for $i=1, \ldots, k$ and we select the best solution. The first cycle of $\operatorname{sol}_{i}$ is formed by the sequence $(0,1, \ldots, i, 0)$ and then each other cycle (except eventually the last) of $\operatorname{sol}_{i}$ has exactly $k$ consecutive vertices (for instance, the second cycle is $(0, i+1, \ldots, i+k, 0))$ and finally, the last cycle is formed by the unvisited vertices (connecting $n$ to the depot 0 ). Denote by $a p x_{i}$ for $i=1, \ldots, k$ the values of the $k$ solutions and by apx the value of the best one.

Consider sol $_{1}, \ldots$, sol $_{k}$; each edge of $T \backslash\{(0,1),(0, n)\}$ appear exactly $(k-1)$ times and each edge $(0, j)$ for $j \neq 1, n$ appears exactly twice. Finally, the edges $(0,1)$ and $(0, n)$ appears exactly $(k+1)$ times. Using Lemma 2 , we deduce: ap $x \leq$ $\frac{1}{k} \sum_{i=1}^{k} a p x_{i} \leq \frac{(k-1)}{k} \operatorname{val}(T)+\frac{2}{k}$ wor $_{V R P}$. By hypothesis, $T$ satisfies: $\operatorname{val}(T) \leq$ $(1-\delta)$ wor $_{T S P}+\delta o p t_{T S P}$ and since it is possible to construct from an optimum solution of VRP a solution of TSP with smaller a value (using the triangle inequality), it follows that $o p t_{T S P} \leq o p t_{V R P}$. Also, by connecting the depot twice with each customer, we can construct from a solution of TSP a solution of VRP with greater value, and therefore wor $_{T S P} \leq w o r_{V R P}$.

Using the previous inequalities we obtain that

$$
a p x \leq \delta \frac{k-1}{k} o p t_{V R P}+\left(1-\delta \frac{k-1}{k}\right) \text { wor }_{V R P}
$$

Since the best known differential approximation algorithm for TSP is $\frac{2}{3}[11$, 14] then the algorithm of Theorem 2 is an $\frac{2}{3} \cdot \frac{k-1}{k}$ differential approximation algorithm for metric $k \mathrm{VRP}$. So, for $k \geq 5$, we obtain a ratio strictly better that the bound produced by the Theorem 1 or by the previous algorithm build on a matching. Now, we will improve all the previous bounds for $k>3$ since we give a $\frac{3}{5}$ (resp., $\frac{2}{3}$ ) differential approximation for METRIC 4VRP (resp., METRIC $k \mathrm{VRP}$ for $k \geq 5$ ).

Theorem 3. METRIC $k \mathrm{VRP}$ is $\min \left\{\frac{2}{3}, \frac{k-1}{k+1}\right\}$ differential approximable.

Proof. Our algorithm works as follow: we compute a minimum weight binary 2-matching $M=\left(C_{1}, \ldots, C_{q}\right)$ on the subgraph induced by $\{1, \ldots, n\}$. Then, for each cycle of it, we produce several solutions and we take the best one among them. These different solutions depend on the size $m_{i}$ of the cycle $C_{i}$ of $M$. 
Without loss of generality, assume that this cycle is described by the sequence $\left(1, \ldots, m_{i}, 1\right)$ with $m_{i} \geq 3$. Let wor $_{i}=2 \sum_{j=1}^{m_{i}} d_{0, j}$.

If $m_{i} \leq k$, then we produce $m_{i}$ solutions sol $_{1}, \ldots$, sol $_{m_{i}}$ where $\operatorname{sol}_{j}$ is obtained just by deleting the edge $(j, j+1)\left(\bmod m_{i}\right)$ and by connecting $j$ and $j+1$ to the depot. Since wor $_{i} \geq d\left(C_{i}\right)$ by the triangle inequality and $m_{i} \geq 3$ we have: $a p x_{i}=\min _{j} d\left(\right.$ sol $\left._{j}\right) \leq \frac{m_{i}-1}{m_{i}} d\left(C_{i}\right)+\frac{1}{m_{i}}$ wor $_{i} \leq \frac{2}{3} d\left(C_{i}\right)+\frac{1}{3}$ wor $_{i}$.

Now, assume that $m_{i}=k p+r$ with $p \geq 1$ and $0 \leq r \leq k-1$. We still produce $m_{i}$ solutions $\operatorname{sol}_{1}, \ldots$, sol $_{m_{i}}$ but in this case in a different way. In order to build the solution $\operatorname{sol}_{j}$, we delete from $C_{i}$ the edge $(j-1, j)$ and the edges $(j-1+r+k \ell, j+r+k \ell)$ for $\ell=1, \ldots, p$ (the indices are taken $\left.\bmod m_{i}\right)$ and we add for each endpoint of these paths, the link with the depot. Note that when $r=1$, the first path is the isolated vertex $j$ and then in this case, we add once again the edge $(0, j)$.

If $r=0$, then we deduce: $a p x_{i} \leq \frac{p(k-1)}{k p} d\left(C_{i}\right)+\frac{p}{k p}$ wor $_{i} \leq \frac{2}{3} d\left(C_{i}\right)+\frac{1}{3}$ wor $_{i}$.

If $r \geq 1$, then we obtain: $a p x_{i} \leq \frac{p(k-1)+r-1}{k p+r} d\left(C_{i}\right)+\frac{p+1}{k p+r} w r_{i} \leq \frac{k-1}{k+1} d\left(C_{i}\right)+$ $\frac{2}{k+1}$ wor $_{i}$.

Finally, since $d(M) \leq o p t_{V R P}$, by taking the minimum between $\frac{2}{3}$ and $\frac{k-1}{k+1}$ and by summing over $i$ the previous inequalities we obtain the expected result.

Since $n \mathrm{VRP}$ and TSP are standard equivalent by using the result of Papadimitriou and Yannakakis [16] we deduce immediately that $n \operatorname{VRP}(1,2)$ has no standard approximation scheme. Also TSP $(1,2)$ has no differential approximation scheme, Monnot et al. [15] but we cannot deduce immediately that $n \operatorname{VRP}(1,2)$ has no differential approximation scheme since $w_{n V R P}$ and wor $_{T S P}$ can be very far. However, we prove in the following a lower bound for the approximation of $n \operatorname{VRP}(1,2)$.

Theorem 4. METRIC $n \operatorname{VRP}(1,2)$ is not $\frac{2219}{2220}-\varepsilon$ differential approximable, for any constant $\varepsilon$, unless $\mathbf{P}=\mathbf{N P}$.

Proof. Since $w_{n V R P} \leq 4 n \leq 4 o p t_{n V R P}$, a $\delta$ differential approximation for $n \operatorname{VRP}(1,2)$ gives a $\delta+4(1-\delta)$ standard approximation for $n \operatorname{VRP}(1,2)$. Using the negative result given in Engebretsen and Karpinski[6]: $\operatorname{TSP}(1,2)$ is not $\frac{741}{740}-$ $\varepsilon$ standard approximable, then we obtain the expected result.

\section{Constrained VRP}

We assume now that each edge is associated with a weight $\ell$ (where $\ell_{i, j} \geq 0$ denotes the cost/time of traversing the edge $(i, j))$ satisfying the triangle inequality, and the solution must satisfy that the total weight on each cycle does not exceed $\lambda$. Note that if we do not assume that $\ell$ is a metric then even deciding whether the problem has any feasible solution is NP-complete.

Theorem 5. Deciding the feasibility of Constrained VRP is NP-complete. 
Proof. In order to prove the NP-hardness, we reduce Hamiltonian $s-t$ Path problem to Constrained VRP. From a graph $G=(V, E)$ with $V=\{1, \ldots, n\}$, we construct a graph $G^{\prime}$ instance of CONSTRAINED VRP by adding a depot vertex 0 . We define the function $l$ as follows: $\ell_{0, s}=\ell_{0, t}=1, \ell_{0, i}=\lambda$, for $i \neq s, t$, $\ell_{i, j}=1$ if $(i, j) \in E$ and $\ell_{i, j}=\lambda$ if $i, j \in\{1, \ldots, n\}$ and $(i, j) \notin E$. Trivially there is a feasible solution for $G^{\prime}$ only if $\lambda \geq n+1$. It is easy to see that Constrained VRP has a feasible solution iff $G$ contains a Hamiltonian path between $s$ and $t$.

Therefore, we assume that $\ell$ satisfies the triangle inequality, and to ensure feasibility we also assume that $2 \ell_{0, i} \leq \lambda$ for $i=1, \ldots, n$. Using similar ideas as those used for $k \mathrm{VRP}$ we can prove:

Theorem 6. Constrained VRP is $\frac{1}{3}$ differential approximable.

Proof. We start with a binary 2-matching as described in Lemma 1 except that the initial graph is not a complete undirected graph $K_{n+1}$ but a partial graph $G^{\prime}$ of it built by deleting the edges $(i, j)$ for $i \neq 0$ and $j \neq 0$ such that $\ell_{0, i}+\ell_{i, j}+$ $\ell_{j, 0}>\lambda$. It is easy to observe that $M$ is still a lower bound of an optimal solution of Constrained VRP. As previously, we work on each cycle of $M$ separately.

First, consider a cycle of $M$ that does not contain the depot, and w.l.o.g. denote its vertices by $(1, \ldots, m)$. The construction depends on the parity of $m$. If $m$ is even, we produce two same solutions $\mathrm{sol}_{1}$ and $\mathrm{sol}_{2}$ of Theorem 1, i.e., $(0,1,2,0),(0,3,4,0), \ldots,(0, m-1, m, 0)$ and $(0, m, 1,0),(0,2,3,0),(0,4,5,0), \ldots$, $(0, m-2, m-1,0)$. Now, if $m$ is odd, we produce $m$ solutions $s^{2} l_{1}, \ldots$, sol $_{m}$ where the solution $\operatorname{sol}_{i}$ for $i=1, \ldots, m$ consist of the cycles $(0, i, 0),(0, i+1, i+$ $2,0),(0, i+3, i+4,0), \ldots,(0, i-2, i-1,0)$ (the indices are taken mod $m)$.

Second, consider a cycle $(0,1, \ldots, m, 0)$ in $M$. If $\sum_{i=1}^{m} w_{i} \leq \lambda$ we don't change it. Otherwise, we have $m \geq 3$ and we produce two solutions $s l_{1}$ and $s o l_{2}$ depending on the parity of $m$. Let us consider the two cases:

Suppose that $m$ is even. Let $s o l_{1}$ consist of the cycles $(0,1,2,0),(0,3,4,0), \ldots$, $(0, m-1, m, 0)$. Let $s_{2} l_{2}$ consist of the cycles $(0,1,0),(0,2,3,0), \ldots,(0, m-$ $2, m-1,0),(0, m, 0)$. Now, suppose that $m$ is odd. Let sol $_{1}$ consist of the cycles $(0,1,0),(0,2,3,0), \ldots,(0, m-1, m, 0)$. Let $s^{2} l_{2}$ consist of the cycles $(0,1,2,0)$, $(0,3,4,0), \ldots,(0, m-2, m-1,0),(0, m, 0)$.

In all cases the solution bad $=(0,1,0), \ldots,(0, n, 0)$ satisfies $\min _{i} \delta\left(\operatorname{sol}_{i}\right) \leq$ $\frac{2}{3} \delta(b a d)$ (this relation holds with equality when each cycle has exactly three vertices and does not contain the depot, since in this case $\delta\left(\operatorname{sol}_{1}\right)+\delta\left(\operatorname{sol}_{2}\right)+$ $\left.\delta\left(\mathrm{sol}_{3}\right)=2 \delta(\mathrm{bad})\right)$. Therefore, by (1), the best of the solutions sol $_{i}$ is a $\frac{1}{3}$ differential approximation.

In Haimovich et al. [9], the authors consider two versions of $k \mathrm{VRP}$ with additional constraint on the length of each cycles. In the first problem that we will call here WEIGHTED $k$ VRP ( $k$ WVRP), each customer has a weight and we want to find a solution such that the total customer weight on each cycle does not exceed $k$. In the second, called in [9] Min Metric Distance, we want to find a solution such that the total distance on each cycle does not exceed a given bound $\lambda$. For each of these two problems, we give a differential reduction preserving approximation scheme from ConstRAINED VRP. 
Lemma 3. $A \delta$ differential approximation solution for CONSTRAINED VRP (respectively, metric case) is also a $\delta$ differential approximation for $k \mathrm{WVRP}$ (respectively, metric case).

Proof. Let $G=(V, E)$ with $d$ and $w$ be an instance of $k$ WVRP. We construct an instance of CONSTRAINED VRP as follows. First we set $\lambda=k$. The graph and the function $d$ are the same whereas the function $\ell$ is defined by: $\ell_{i, j}=\frac{w_{i}+w_{j}}{2}$ where we assume that $w_{0}=0$. This function satisfies the triangle inequality. Moreover, let $C$ be a cycle linking the depot to a subset of customers. We have $\sum_{q \in C} w_{q} \leq k$ iff $\sum_{(i, j) \in C} \ell_{i, j} \leq \lambda$.

Corollary 1. $k \mathrm{WVRP}$ is $\frac{1}{3}$ differential approximable.

Min Metric Distance is a particular case of Metric Constrained VRP where the function $\ell$ is exactly the function $d$. Thus, from Theorem 6 we deduce the corollary:

Corollary 2. Min MetriC Distance is $\frac{1}{3}$ differential approximable.

\section{Min-Sum EkTSP}

It is easy to see that MiN-SUM E $k$ TSP is differential equivalent to METRIC Min-Sum E $k$ TSP since the number of edges in every solution is the same (like in the TSP case). Hence, we add a constant to all the edge lengths and achieve the triangle inequality without affecting the best and worst solutions.

Theorem 7. Metric Min-Sum E $k$ TSP is $\frac{2}{3}$ differential approximable, $\forall k \geq 1$.

Proof. Add to every edge incident with the depot a parallel copy. Compute a minimum binary f-matching $M$ on $G$ where $f(0)=2 k$ and $f(v)=2$ for $v \in V \backslash\{0\}$. Compute by using an algorithm of Hassin and Khuller [11] or Monnot [14] a solution $C^{\prime}$ for TSP on the subgraph $G^{\prime}$ of $G$ induced by $V^{\prime}=$ $V \backslash\left(\cup_{i=1}^{k-1} V\left(C_{i}\right)\right) \cup\{0\}$, where $C_{1}, \ldots, C_{k}$ are the cycles of $M$ containing the depot 0 . The approximate solution sol is composed of $C^{\prime}$ and the cycles $C_{1}, \ldots, C_{k-1}$. See Figure 3. Since $M$ is an minimum binary f-matching $M$ on $G$ then $M^{\prime}=M \backslash$ $\left(\cup_{i=1}^{k-1} C_{i}\right)$ is an optimum binary 2-matching on $G^{\prime}$. We denote by $r=\sum_{i=1}^{k-1} d\left(C_{i}\right)$; the TSP algorithm gives a solution satisfying val $\leq \frac{2}{3} d\left(M^{\prime}\right)+\frac{1}{3}$ wor $_{T S P}\left(G^{\prime}\right)$. Since $\operatorname{wor}_{k T S P}(G) \geq$ wor $_{T S P}\left(G^{\prime}\right)+r$ and $\operatorname{opt}_{k T S P}(G) \geq d(M)$, we deduce that the value of sol satisfies: $a p x=v a l+r \leq \frac{2}{3}\left[d\left(M^{\prime}\right)+r\right]+\frac{1}{3}\left[\operatorname{wor}_{T S P}\left(G^{\prime}\right)+r\right] \leq$ $\frac{2}{3} \operatorname{opt}_{k T S P}(G)+\frac{1}{3}$ wor $_{k T S P}(G)$

Theorem 8. Unless $\mathbf{P}=\mathbf{N P}$, Min-Sum $\operatorname{E} k \operatorname{TSP}(1,2)$ has no standard and differential approximation scheme.

Proof. We reduce Min TSP PATH $(1,2)$ on Hamiltonian cubic graphs to MiNSum E2TSP. From a graph $G=(V, E)$ on $n$ vertices, we construct a graph $G^{\prime}$ instance of Min-Sum E2TSP. $G^{\prime}$ consists of two copies of $G$ and a vertex 0 (the 


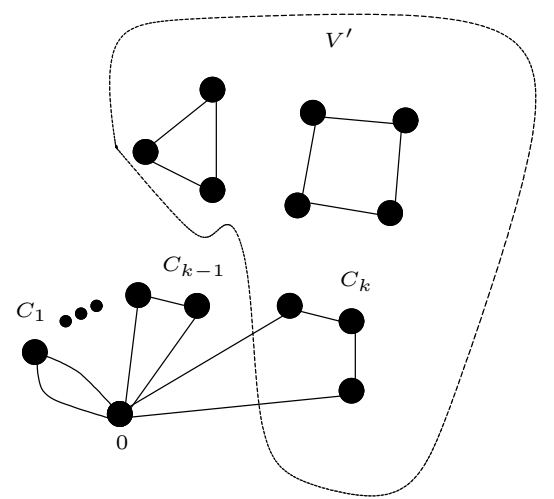

$M$

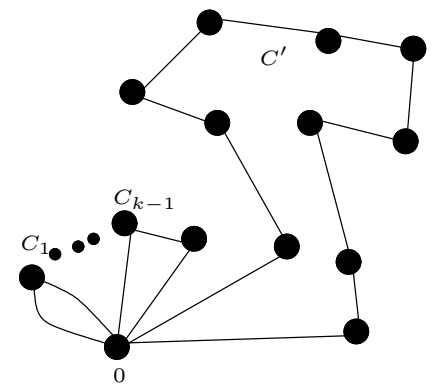

sol

Fig. 3. $M$ and sol

depot). Within a copy, the edges have the same distance as in $G ; d_{0, i}=1$, for each vertex $i$ in one of the two copies; $d_{i, j}=2$ if $i$ and $j$ are vertices in different copies. We have $\operatorname{opt}\left(G^{\prime}\right)=2 \operatorname{opt}(G)+4$ and $\operatorname{wor}\left(G^{\prime}\right)=2 \operatorname{wor}(G)+4$. Given a solution $S$ of $G^{\prime}$ with two cycles, we can transform it in another one $S^{\prime}$ that contains exactly two cycles $\left(0, P_{1}, 0\right),\left(0, P_{2}, 0\right)$, each of these two paths are contained in a copy of $G$ and with a better value. The idea for doing this is to remove the edges between the two copies and to replace them by the missing edges in the two copies. We consider as solution for $G$ the path with the smallest value among the two. So, $\operatorname{val}=\min \left\{\operatorname{val}\left(P_{1}\right), \operatorname{val}\left(P_{2}\right)\right\} \leq \frac{\operatorname{val}\left(P_{1}\right)+\operatorname{val}\left(P_{2}\right)}{2}=\frac{\operatorname{val}\left(S^{\prime}\right)-4}{2} \leq \frac{\operatorname{val}(S)-4}{2}$. Since $\operatorname{opt}(G)=\frac{\operatorname{opt}\left(G^{\prime}\right)}{2}-2$ and $\operatorname{wor}(G)=\frac{\operatorname{wor}\left(G^{\prime}\right)}{2}-2$ then a $\delta$ differential approximation of Min-Sum E2TSP gives a $\delta$ differential approximation for MiN TSP PATH $(1,2)$ on Hamiltonian and cubic graphs and the conclusion follows.

\section{References}

1. G. Ausiello, A. D'Atri and M. Protasi, "Structure preserving reductions among convex optimization problems," Journal of Computing and System Sciences 21 (1980) 136-153.

2. C. Bazgan, Approximation of optimization problems and total function of NP, Ph.D. Thesis (in French), Université Paris Sud (1998).

3. M. Bellmore and S. Hong, "Transformation of Multi-salesmen Problem to the Standard Traveling Salesman Problem," Journal of the Association for Computing Machinery 21 (1974) 500-504.

4. W.J. Cook, W.H. Cunningham, W.R. Pulleyblank, and A. Schrijver Combinatorial Optimization John Wiley \& Sons Inc New York 1998 (Chapter 5.5).

5. M. Demange and V. Paschos, "On an approximation measure founded on the links between optimization and polynomial approximation theory," Theoretical Computer Science 158 (1996) 117-141. 
6. L. Engebretsen and M. Karpinski, "Approximation hardness of TSP with bounded metrics," http://www.nada.kth.se/ enge/papers/BoundedTSP.pdf

7. M. R. Garey and D. S. Johnson, "Computers and intractability. A guide to the theory of NP-completeness," Freeman, C.A. San Francisco (1979).

8. M. Haimovich and A. H. G. Rinnooy Kan, "Bounds and heuristics for capacitated routing problems," Mathematics of Operations Research 10 (1985) 527-542.

9. M. Haimovich, A. H. G. Rinnooy Kan and L. Stougie, "Analysis of Heuristics for Vehicle Routing Problems," in Vehicle Routing Methods and Studies, Golden, Assad editors, Elsevier (1988) 47-61.

10. D. Hartvigsen, Extensions of Matching Theory. Ph.D. Thesis, Carnegie-Mellon University (1984).

11. R. Hassin and S. Khuller, "z-approximations," Journal of Algorithms 41 (2001) 429-442.

12. D. G. Kirkpatrick and P. Hell, "On the completeness of a generalized matching problem," Proc. of the 10th ACM Symposium on Theory and Computing (1978) 240-245.

13. C-L. Li, D. Simchi-Levi and M. Desrochers, "On the distance constrained vehicle routing problem," Operations Research 40 4(1992) 790-799.

14. J. Monnot, "Differential approximation results for the traveling salesman and related problems," Information Processing Letters 82 (2002) 229-235.

15. J. Monnot, V. Th. Paschos and S. Toulouse, "Differential Approximation Results for the Traveling Salesman Problem with Distances 1 and 2," Proc. FCT (2001) 275-286.

16. C. Papadimitriou and M. Yannakakis, "The traveling salesman problem with distances one and two," Mathematics of Operations Research 18(1) (1993) $1-11$.

17. S. Sahni and T. Gonzalez, "P-complete approximation problems" Journal of the Association for Computing Machinery 23 (1976) 555-565.

18. E. Zemel, "Measuring the quality of approximate solution to zero-one programming problems," Mathematical Operations Research 6(1981) 319-332. 